TUA3.3

2:15 pm - 2:30 pm

\title{
Design of high-Q photonic crystal optical cavities through group-theoretical and Fourier space analyses
}

\author{
Kartik Srinivasan, Paul E. Barclay, and Oskar Painter \\ Department of Applied Physics, California Institute of Technology, Pasadena, CA \\ 91125, USA. \\ phone: (626) 395-6269, fax: (626) 795-7258, e-mail: kartik@caltech.edu
}

\begin{abstract}
Group-theoretical and Fourier space analyses are used to design low loss photonic crystal cavities. Experimental results verifying the group theory predictions are presented, along with finite-difference time-domain calculations of recent designs with predicted quality factors exceeding $10^{5}$.

(C) 2003 IEEE LEOS

OCIS codes: (230.5750) Resonators; (140.5960) Semiconductor Lasers
\end{abstract}

Photonic crystal (PC) defect cavities [1] are promising elements for a number of devices and experiments in the optical sciences, including low threshold semiconductor lasers and resonators for probing coherent electron-photon interactions. These applications take advantage of the cavities' wavelength-scale size, but also require them to have minimal radiation losses, with a quality factor $(Q)$ of $10^{4}$ or $10^{5}$. We consider the design of these cavities in Fourier space, developing a set of simple rules for obtaining high- $Q$ modes. Prospective modes that satisfy these rules are determined by a group-theory based analysis [2] that gives approximate forms for donor and acceptor modes localized to defects at high symmetry points within hexagonal and square lattice PCs. The predictive power of the symmetry analysis is confirmed by finite-difference timedomain (FDTD) calculations and experimental data in the form of photoluminescence (PL) measurements of fabricated devices. With these results as a baseline, we present FDTD results that predict $Q s$ in excess of $10^{5}$ for defect geometries designed according to our Fourier space guidelines [3]. In addition, efficient fiber-based methods for coupling light into and out of these planar PC cavities are discussed.

The group theory analysis consists of two steps. We first generate approximate forms for the band-edge states at the high symmetry points within the first Brillouin zone (IBZ) of the lattice, and then use these states as a basis to generate approximate forms for localized defect modes. The field pattern for a dipolelike donor mode in a hexagonal lattice generated by this analysis is given in figure 1(a), while figure 1(b) shows the results of FDTD calculations for the same mode. This close correspondence is complemented by experimental results in the form of a PL spectrum from a microfabricated PC cavity, as given in Figure 1(c). As we shall discuss, not only are these measurements well-fit by FDTD simulations, all of the modes present within the spectrum are accounted for by the group theory analysis.

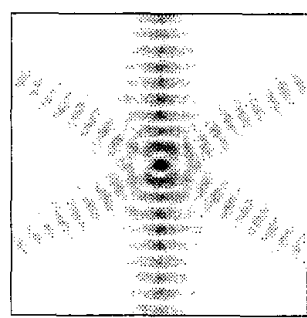

(a) Group theory.

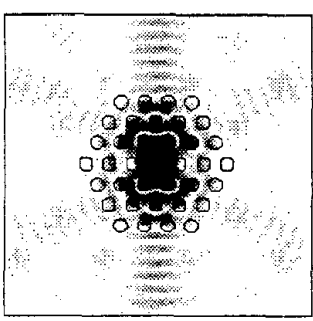

(b) FDTD.

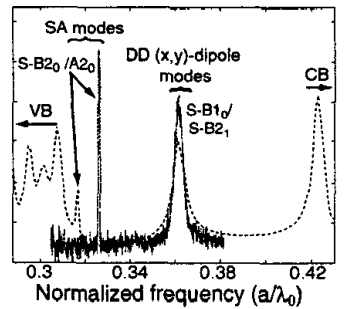

(c) PL measurements.

Fig. 1. (a) Electric field amplitude of the hexagonal lattice $\hat{x}$-dipole mode generated by the symmetry analysis. (b) FDTD-generated field pattern. (c) PL and FDTD spectra of a single missing air hole defect cavity. FDTD simulation results are shown as a dashed line.

Having used group theory as a tool for understanding the modal structure of PC defect cavities, we seek to create defect geometries that sustain high- $Q$ modes. Defect modes in two-dimensional PC slab waveguides have in-plane losses that are determined by the size and angular extent of the photonic bandgap in momentum space, while vertical radiation losses result when the mode's in-plane momentum components are insufficient 
to support waveguiding, and lie within the light cone of the waveguide cladding. To reduce the presence of these small momentum components, we seek modes that are odd about mirror planes normal to the direction of the mode's dominant Fourier components, eliminating the DC $\left(\mathbf{k}_{\perp}=\mathbf{0}\right)$ part of the field. Modes of the appropriate symmetry are determined by the aforementioned group-theory based analysis.

FDTD simulations show improved $Q$ factors for defect modes of this prescribed symmetry. Further improvements to both the in-plane and vertical $Q s$ are achieved by considering a Fourier space picture of modal couplings that lead to loss. The results of this analysis indicate that the quantity $\widetilde{\Delta \eta}\left(\mathbf{k}_{\perp}\right)$, the Fourier transformed perturbation to the photonic lattice $(\eta=1 / \epsilon)$ due to the defect, couples Fourier components between the basis modes of the system [4]. Thus, by appropriately modifying the lattice to tailor this quantity, we reduce couplings that lead to in-plane and vertical leakage. Doing so results in the square lattice design shown in Figure 2(a). Figure 2(b)-(d) shows the magnetic field amplitude and Fourier transformed dominant electric field component for this mode, indicating the degree to which power has been removed from the cladding light cone. $Q_{\perp} \approx 1.3 * 10^{5}$ for this design, with $Q_{\text {tot }} \approx 9.8 * 10^{4}$. The design rules described above have also been applied to hexagonal lattice PC's, with FDTD-determined $Q$ values exceeding $10^{5}$. Representative geometries and field patterns for such designs are shown in Figure 3(a)-(d).

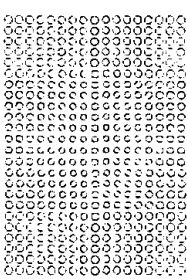

(a) Lattice

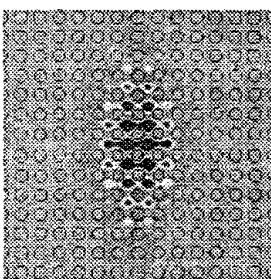

(b) $|\mathrm{B}|$

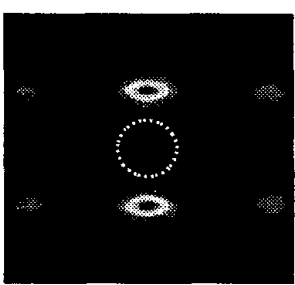

(c) $\left|\widetilde{\mathbf{E}}_{x}\right|$

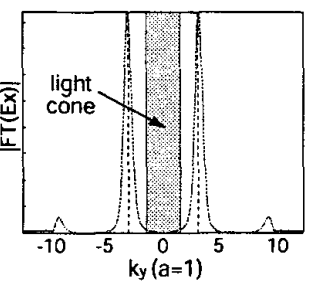

(d) $\left|\widetilde{\mathbf{E}}_{x}\right|$ vs. $k_{y}$ axis.

Fig. 2. Graded square lattice and its field characteristics.

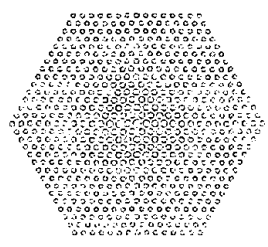

(a) Lattice

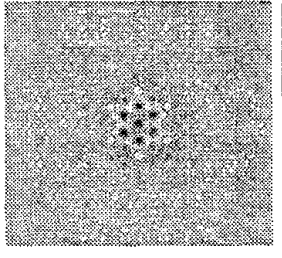

(b) $\mid \mathbf{B}$

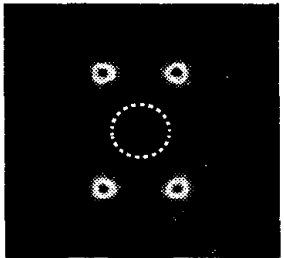

(c) $\left|\widetilde{\mathbf{E}}_{x}\right|$

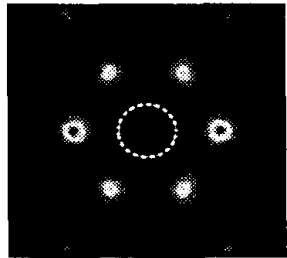

(d) $\left|\widetilde{\mathbf{E}}_{y}\right|$

Fig. 3. Graded hexagonal lattice and its field characteristics.

In addition to outlining the methods and results described above, we will discuss a number of important topics that are also under consideration, such as efficient coupling of the cavities with PC waveguides and schemes by which cavity-waveguide systems can be passively probed with tapered optical fibers. Finally, our recent progress in the fabrication and characterization of these devices will be presented.

\section{References}

1. S. Noda, A. Chutinan, and M. Imada, "Trapping and emission of photons by a single defect in a photonic bandgap structure," Nature 407, 608-610 (2000).

2. O. Painter and K. Srinivasan, "Localized defect states in two-dimensional photonic crystal slab waveguides: a simple model based upon symmetry analysis," accepted for publication in Phys. Rev. B.

3. K. Srinivasan and O. Painter, "Momentum space design of high-Q photonic crystal optical cavities," Opt. Express 10, 670-684 (2002).

4. O. Painter, K. Srinivasan, and P. E. Barclay, "A Wannier-like Equation for the Resonant Optical Modes of Locally Perturbed Photonic Crystals," submitted to Phys. Rev. B, February 2003. 